\title{
Isolated hypogonadotropic hypogonadism with SOX2 mutation and anophthalmia/microphthalmia in offspring
}

\author{
Zornitza Stark $^{1}$, Rebecca Storen ${ }^{2,5}$, Bruce Bennetts ${ }^{3,5}$, Ravi Savarirayan ${ }^{1,4}$ and Robyn V Jamieson ${ }^{\star 2,5}$ \\ Isolated hypogonadotropic hypogonadism (IHH) is a genetically heterogeneous condition in which patients frequently require \\ assisted reproduction to achieve fertility. In patients with IHH who are otherwise well, no particular increased risk of congenital \\ anomalies in the resultant offspring has been highlighted. Heterozygous mutations in SOX2 are the commonest single-gene \\ cause of anophthalmia/microphthalmia (A/M) and sometimes result in pituitary abnormalities. We report a family with a novel \\ frameshift mutation in the SOX2 transactivation domain, p.Gly280AlafsX91, resulting in bilateral anophthalmia and subtle \\ endocrinological abnormalities in a male sibling, and unilateral microphthalmia in a female sibling. The mutation is present in \\ their mother who has IHH, but has no eye disorders or other anomalies. She underwent assisted reproduction to achieve fertility. \\ This report has important implications for the evaluation of patients with $\mathrm{IHH}$, particularly in the setting of planned infertility \\ treatment.
}

European Journal of Human Genetics (2011) 19, 753-756; doi:10.1038/ejhg.2011.11; published online 16 February 2011

Keywords: hypogonadotropic hypogonadism; SOX2; anophthalmia; microphthalmia

\section{INTRODUCTION}

Isolated hypogonadotropic hypogonadism (IHH) is caused by impaired release of gonadotropins (follicle-stimulating hormone (FSH) and luteinizing hormone (LH)) in the setting of otherwise normal anterior pituitary anatomy and function. The resulting hypogonadism typically manifests as incomplete development of secondary sexual characteristics. IHH has an incidence of 1:10000-1:86000, ${ }^{1}$ although the true prevalence is difficult to determine in the absence of detailed reproductive phenotyping in large unselected populations. In approximately two-thirds of patients, IHH is associated with anosmia (Kallmann syndrome). IHH is a genetically heterogeneous condition, and mutations in a number of genes have been identified to date, including KAL1, ${ }^{2}$ FGFR1, ${ }^{3}$ PROKR2, ${ }^{4}$ PROK2, ${ }^{4}$ GNRHR, ${ }^{5}$

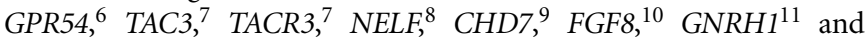
WDR $11 .^{12}$ However, mutations in these genes only account for a small proportion of affected patients, and in the majority of cases, the aetiology is unknown.

SOX2 is a member of the sex-determining region of the $\mathrm{Y}$ chromosome-related high-mobility group box (SOX) family of transcription factors. ${ }^{13}$ Expression of SOX2 is critical for human eye development, with heterozygous mutations in this gene found in approximately $10 \%$ of cases of anophthalmia/microphthalmia (A/M). ${ }^{14-17}$ SOX2 has also been shown to have a key role in the development of other tissues, notably the pituitary, forebrain, gastrointestinal and urogenital systems. ${ }^{14,15}$

We report a family with $\mathrm{A} / \mathrm{M}$ in siblings, who were born to a mother with $\mathrm{IHH}$ and no ocular anomalies. A novel frameshift mutation in SOX2 was identified in the siblings, and this was also present in the mother.

\section{PATIENTS AND METHODS}

Clinical findings

The couple in this family (I.1 and I.2, Figure 1a) was reviewed after their two children (II.1 and II.2, Figure 1a) were born with A/M following assisted reproduction. The couple were Caucasian and non-consanguineous with no known eye disorders in themselves or in their families. The mother, I.2 (Figure 1a, Table 1) previously had an endocrine investigation at the age of 18 years because of primary amenorrhoea and limited development of secondary sexual characteristics. Her investigations revealed low FSH $3.5 \mathrm{mIU} / \mathrm{ml}(2.0-16.0), \mathrm{LH}<3.0 \mathrm{mIU} / \mathrm{ml}(4.0-20.0)$ and oestradiol $11 \mathrm{pg} / \mathrm{ml}$ (prepubertal 10-25). Prolactin and thyroid function tests, and a computed tomography scan of the pituitary and hypothalamus were normal. Patient I.2 was normally grown, had a normal sense of smell and was not dysmorphic. Subsequent ophthalmological examination, including dilated fundal examination, was normal. She was diagnosed with IHH.

The couple's first child, II.1 (Figure 1a, Table 1), was conceived through gonadotropin ovulation induction (75 IU Humegon, NV Organon, Oss, The Netherlands, and 75 IU Puregon, Schering-Plough, Kenilworth, NJ, USA) and was delivered at 37 weeks gestation by Caesarean section for maternal pre-eclampsia. Bilateral anophthalmia was noted at birth, with deeply set orbits, and normal eyelids and eyelashes. An apnoeic episode accompanied by abnormal movements occurred at the age of $17 \mathrm{~h}$ in the setting of transient hypocalcaemia, and he was treated with anti-convulsants for a brief period. He was not dysmorphic, had descended testes and a normal phallus.

Birth parameters in patient II.1 were weight $3 \mathrm{~kg}$, length $47 \mathrm{~cm}$ and head circumference $34.5 \mathrm{~cm}$, all on the 50th centile. Cortisol and growth hormone

${ }^{1}$ Genetic Health Services Victoria, and Murdoch Children's Research Institute, Melbourne, Victoria, Australia; 'Eye Genetics Research Group, The Children's Hospital at Westmead, Children's Medical Research Institute, Save Sight Institute, University of Sydney, Sydney, New South Wales, Australia; ${ }^{3}$ Department of Molecular Genetics, The Children's Hospital at Westmead, Sydney, New South Wales, Australia; ${ }^{4}$ Department of Paediatrics, University of Melbourne, Melbourne, Victoria, Australia; ${ }^{5}$ Sydney Medical School, University of Sydney, Sydney, New South Wales, Australia

*Correspondence: Associate Professor RV Jamieson, The Children's Hospital at Westmead and Children's Medical Research Institute, Hawkesbury Road, Westmead, Sydney, New South Wales 2145, Australia. Tel: +61 2 96872800; Fax: +61 2 96872120; E-mail: rjamieson@cmri.usyd.edu.au

Received 4 August 2010; revised 11 January 2011; accepted 12 January 2011; published online 16 February 2011 
a

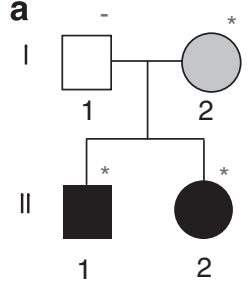

b i

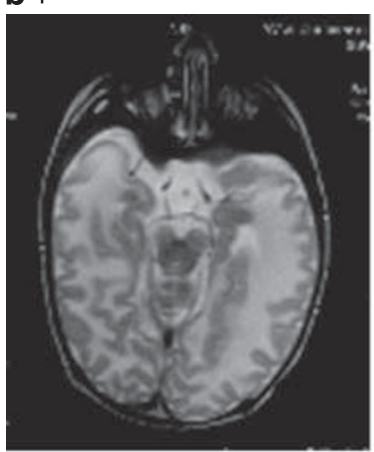

iii

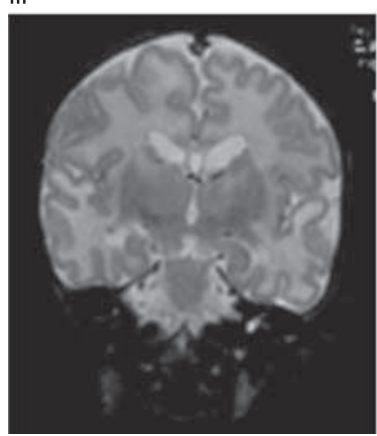

Isolated hypogonadotropic hypogonadism

Anophthalmia/microphthalmia ii

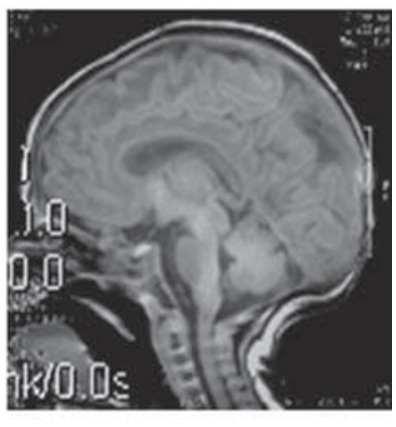

e d

I.1

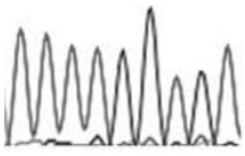

II.1

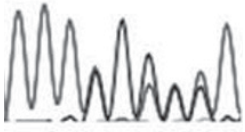

II.2

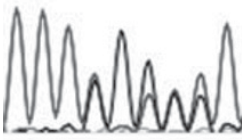

I.2a

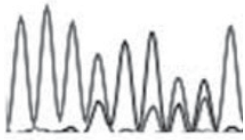

I. $2 \mathrm{~b}$

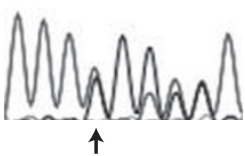

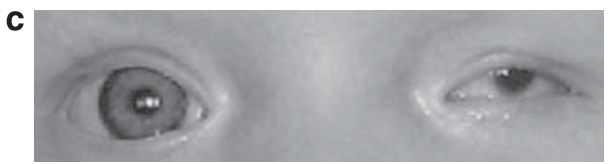

Figure 1 Pedigree, clinical features and SOX2 mutation in family. (a) In this pedigree, individuals with the SOX2 mutation, p.Gly280AlafsX91, are identified by an asterisk. The individual without the SOX2 mutation is identified by the minus sign. Individuals identified by a black symbol (II.1 and II.2) have bilateral or unilateral anophthalmia/microphthalmia. Patient II.1 also had growth hormone deficiency. The individual identified by the grey symbol (I.2) has isolated hypogonadotropic hypogonadism with no eye disorder or other anomalies. (b) Magnetic resonance images of the brain and orbits in patient II.1. (i) Transverse section showing complete absence of ocular structures and optic nerves. (ii) Sagittal section demonstrating normal pituitary and corpus callosum. (iii) Coronal section demonstrating asymmetric hippocampi with the right hippocampus appearing dysplastic and under rotated. (c) Left microphthalmia in patient II.2. (d) Direct sequencing of PCR product from affected children II.1 and II.2 in the family showed heterozygous c.837delC (NM_003106). This was present in the mother, I.2, with an apparently lower level of mutant allele in her DNA sample extracted from blood (I.2a), but was clearly present in her DNA sample extracted from buccal cells (I.2b). The mutation was absent from control sequence and the father (I.1). (e) The c.837delC mutation results in a frameshift mutation, p.Gly280AlafsX91, in the $\mathrm{C}$ terminal end of the transactivation domain of SOX2.

Table 1 Ocular, cerebral and pituitary phenotypes of c.837delC SOX2 family members

\begin{tabular}{|c|c|c|c|c|}
\hline & Eye morphology & Fundus & Neuroimaging results & Pituitary function \\
\hline 1.2 & Normal & Normal & Normal (CT) & Isolated hypogonadotropic hypogonadism \\
\hline II.1 & Bilateral anophthalmia & $\mathrm{N} / \mathrm{A}$ & Normal pituitary, Dysplastic right hippocampus (MRI) & Transient growth and GH disturbance \\
\hline 11.2 & $\begin{array}{l}\text { Left: severe microphthalmia } \\
\text { Right: normal }\end{array}$ & $\begin{array}{l}\text { Left: retinal coloboma } \\
\text { Right: normal }\end{array}$ & Not done & Clinically normal \\
\hline
\end{tabular}

levels were equivocal at 3 months, and a glucagon stimulation test demonstrated a subnormal peak growth hormone response $(11 \mathrm{mU} / \mathrm{l})$ and a normal cortisol response $(723 \mathrm{nmol} / \mathrm{l})$. This was accompanied by a period of suboptimal growth. At the age of 1 year, weight was $8.6 \mathrm{~kg}$ (10th centile), length was $71 \mathrm{~cm}$ (2nd centile) and head circumference was $43 \mathrm{~cm}$ (2nd centile). Growth has subsequently normalised, and at the age of 8 years, all parameters 
were between 10 and 25th centiles. Pituitary function is monitored on a yearly basis, and is currently normal.

In patient II.1, MRI scan of the brain and orbits was performed at 12 months of age. No eye remnants were visible and the optic nerves were absent (Figures 1b, i). The corpus callosum was intact, and the pituitary gland was structurally normal and normally located (Figure 1b, ii), whereas the right hippocampus appeared dysplastic and under rotated (Figure 1b, iii). Auditory brainstem response testing in the newborn period and pure tone audiometry at the age of 9 years were normal. Psychomotor development is progressing normally at the age of 9 years, apart from a minor delay in the attainment of early gross motor skills consistent with visual impairment.

The couple's second child, II.2 (Figure 1a, Table 1), was conceived using in vitro fertilisation, and was delivered at term, following an uncomplicated pregnancy, with a birth weight of $2.7 \mathrm{~kg}$ (10th centile). She was noted to have left-sided moderate microphthalmia, with a large retinal coloboma, involving the central vision areas and the optic disc (Figure 1c). The other eye is structurally and functionally normal. There were no associated abnormalities or dysmorphic features. When last assessed at the age of 8 months, she was growing normally, with all parameters between 25 and 50th centile, and had normal development.

\section{Mutation detection}

Whole-blood samples were obtained from all patients, and a buccal cell sample was obtained from patient I.2. Genomic DNA was extracted for gene amplification. The coding exon and adjacent flanking regions of SOX2 (GenBank accession number NM_003106) were amplified by PCR (primers available on request). The PCR product was purified and sequenced bidirectionally using standard techniques. Written informed consent was obtained from the patients, in accordance with ethics approval from the Children's Hospital at Westmead Ethics Committee.

\section{RESULTS}

\section{Novel SOX2 mutation}

We identified a novel heterozygous mutation in the SOX2 gene in child II.1 in this family. Genomic DNA analysis revealed a C nucleotide deletion at position 837 in the SOX2 gene (c.837delC) (Figure 1d). Sequencing in other family members revealed that the affected sibling (II.2) was heterozygous for the same mutation. The mother's sequencing results from lymphocyte DNA showed presence of this mutation, but at an apparently lower level compared with her children affected with ocular anomalies in three independent sequencing reactions (Figure 1d, I.2a). A buccal sample was obtained from the mother and this showed clear presence of the mutant allele (Figure 1d, I.2b). The sequencing results in the mother suggested the possible presence of mosaicism, although they could also be due to sequencing artefacts that can occur in double-stranded sequencing. We were unable to confirm semi-quantitatively whether mosaicism might be present, as the mutation did not create a new restriction enzyme site, and attempts at capillary electrophoresis of fluorescently labelled PCR products as well as allele-specific PCR did not allow conclusive results because of the $1 \mathrm{bp}$ deletion in a GC-rich region. The father's sequencing results were normal.

The one-nucleotide deletion predicts a frameshift mutation p.Gly280AlafsX91, which leads to abnormality of the protein in the $\mathrm{C}$ terminal end of the transactivation domain. The mutation predicts that the normal 317AA SOX2 protein is changed to a 369AA with 90 abnormal $\mathrm{C}$ terminal residues (Figure 1e).

\section{DISCUSSION}

We report a family with a novel frameshift mutation in the SOX2 transactivation domain, resulting in bilateral anophthalmia and subtle pituitary dysfunction in a male sibling and unilateral microphthalmia in a female sibling. The mutation is also present in their mother who has $\mathrm{IHH}$ and underwent assisted reproduction to achieve fertility.

This is the first report of IHH occurring in the absence of eye involvement in a SOX2 mutation patient. Previous patients reported with SOX2 mutations and with hypogonadotropic hypogonadism have had severe ocular anomalies and anterior pituitary hypoplasia. ${ }^{18,19}$ One patient with unilateral anophthalmia and IHH without structural pituitary abnormality is described. ${ }^{20}$ Mutations in GNRHR, GNRH1, GPR54, TAC3 and TACR3 have previously been identified in IHH patients without associated anomalies. ${ }^{5-7,11}$ The findings in the mother in this family add SOX2 mutation as another possible cause of IHH in an otherwise normal individual.

The affected child in this family with bilateral anophthalmia (II.1, Figure 1a, Table 1) had transient growth hormone deficiency in the absence of pituitary hypoplasia on cerebral imaging. Anophthalmia/ microphthalmia patients with heterozygous SOX2 mutations and pituitary hormone deficiencies usually have associated anterior pituitary hypoplasia. ${ }^{19}$ SOX2 is expressed in the developing hypothalamus and anterior pituitary in mice and humans. The functional pituitary abnormality in our patient, in the absence of anterior pituitary hypoplasia, indicates that SOX2 could be involved independently at multiple levels in development of the hypothalamo-pituitary axis. This emphasises the need for clinical monitoring and investigation of the hypothalamo-pituitary axis in SOX2 mutation patients, even in the absence of pituitary hypoplasia on imaging.

The ocular features of bilateral anophthalmia in one sibling and unilateral microphthalmia with retinal coloboma in the other, in this family, are consistent with the variable phenotypic ocular features that can be seen associated with SOX2 mutations within families. ${ }^{21,22}$ The observed spectrum also includes normal eye development in heterozygote $^{23}$ and mosaic ${ }^{17,23-25} /$ queried mosaic ${ }^{26}$ heterozygote SOX2 mutation patients, as in the mother in this case. In addition to pituitary dysfunction, a substantial proportion of SOX2 mutation patients have other cerebral abnormalities as seen in patient II.1 in this report. Other known extra-ocular features, such as oesophageal abnormalities, sensorineural hearing loss and male urogenital abnormalities ${ }^{14,17}$ were not present in our patients.

Children with $\mathrm{A} / \mathrm{M}$ and SOX2 mutations born to mothers with SOX2 mutation mosaicism/queried mosaicism are reported. ${ }^{17,23-26}$ These mothers have all been phenotypically normal. Our case is the first report of one such mother having IHH. Despite the possibility of mosaicism in the cases reported in the literature as well as our case, the mutations have been detectable on routine sequencing of DNA extracted from a peripheral blood sample in all but one case. Management of $\mathrm{IHH}$ in patients without associated anomalies frequently includes gonadotropin therapy and assisted reproductive technologies to achieve fertility. In cases of $\mathrm{IHH}$, which are associated with autosomal recessive inheritance and mutations in GNRHR, GNRH1, GPR54, TAC3 and TACR3, gonadotropin therapy would not be expected to be associated with an increased risk of a child with a birth abnormality. However, our report shows that the IHH patient with a SOX2 mutation, faces an up to $50 \%$ risk of a child with $\mathrm{A} / \mathrm{M}$ and other anomalies associated with SOX2 mutation. Most SOX2 mutations have been ascertained through the study of patients with ocular abnormalities, and it is not known what proportion of patients with IHH may harbour SOX2 mutations. As this family demonstrates, with the advent of assisted reproductive technology, IHH is no longer a barrier to reproduction. SOX2 testing in IHH has important implications for risk counselling, particularly in the setting of infertility treatment. 


\section{CONFLICT OF INTEREST}

The authors declare no conflict of interest.

\section{ACKNOWLEDGEMENTS}

We thank the family for their participation in the research. We thank Scott Cohen and Katrina Slater for technical advice and assistance. We thank Simone Mandelstam for MRI scan review. We acknowledge support from the Ophthalmic Research Institute of Australia.

1 Seminara SB, Hayes FJ, Crowley Jr WF: Gonadotropin-releasing hormone deficiency in the human (idiopathic hypogonadotropic hypogonadism and Kallmann's syndrome): pathophysiological and genetic considerations. Endocr Rev 1998; 19: 521-539.

2 Franco B, Guioli S, Pragliola A et al: A gene deleted in Kallmann's syndrome shares homology with neural cell adhesion and axonal path-finding molecules. Nature 1991; 353: 529-536.

3 Dode C, Levilliers J, Dupont JM et al: Loss-of-function mutations in FGFR1 cause autosomal dominant Kallmann syndrome. Nat Genet 2003; 33: 463-465.

4 Dode C, Teixeira L, Levilliers J et al: Kallmann syndrome: mutations in the genes encoding prokineticin-2 and prokineticin receptor-2. PLoS Genet 2006; 2: e175.

5 de Roux N, Young J, Misrahi M et al: A family with hypogonadotropic hypogonadism and mutations in the gonadotropin-releasing hormone receptor. N Engl J Med 1997; 337: 1597-1602.

6 de Roux N, Genin E, Carel JC, Matsuda F, Chaussain JL, Milgrom E: Hypogonadotropic hypogonadism due to loss of function of the KiSS1-derived peptide receptor GPR54. Proc Natl Acad Sci USA 2003; 100: 10972-10976.

7 Topaloglu AK, Reimann F, Guclu M et al: TAC3 and TACR3 mutations in familial hypogonadotropic hypogonadism reveal a key role for Neurokinin B in the central control of reproduction. Nat Genet 2009; 41: 354-358.

8 Miura K, Acierno Jr JS, Seminara SB: Characterization of the human nasal embryonic LHRH factor gene, NELF, and a mutation screening among 65 patients with idiopathic hypogonadotropic hypogonadism (IHH). J Hum Genet 2004; 49: 265-268.

$9 \mathrm{Kim}$ HG, Kurth I, Lan F et al: Mutations in CHD7, encoding a chromatin-remodeling protein, cause idiopathic hypogonadotropic hypogonadism and Kallmann syndrome. Am J Hum Genet 2008; 83: 511-519.

10 Falardeau J, Chung WC, Beenken A et al: Decreased FGF8 signaling causes deficiency of gonadotropin-releasing hormone in humans and mice. J Clin Invest 2008; 118: 2822-2831.

11 Bouligand J, Ghervan C, Tello JA et al: Isolated familial hypogonadotropic hypogonadism and a GNRH1 mutation. N Engl J Med 2009; 360: 2742-2748.
12 Kim HG, Ahn JW, Kurth I et al: WDR11, a WD protein that interacts with transcription factor EMX1, is mutated in idiopathic hypogonadotropic hypogonadism and Kallmann syndrome. Am J Human Genet 2010; 87: 465-479.

13 Stevanovic M, Zuffardi O, Collignon J, Lovell-Badge R, Goodfellow P: The cDNA sequence and chromosomal location of the human SOX2 gene. Mamm Genome 1994; 5: 640-642.

14 Fantes J, Ragge NK, Lynch SA et al: Mutations in SOX2 cause anophthalmia. Nat Genet 2003; 33: 461-463.

15 Ragge NK, Lorenz B, Schneider A et al: SOX2 anophthalmia syndrome. Am J Med Genet A 2005; 135: 1-7; discussion 8.

16 Bakrania P, Robinson DO, Bunyan DJ et al: SOX2 anophthalmia syndrome: 12 new cases demonstrating broader phenotype and high frequency of large gene deletions. Br J Ophthalmol 2007; 91: 1471-1476.

17 Schneider A, Bardakjian T, Reis LM, Tyler RC, Semina EV: Novel SOX2 mutations and genotype-phenotype correlation in anophthalmia and microphthalmia. Am J Med Genet $A$ 2009; 149A: 2706-2715.

18 Kelberman D, de Castro SC, Huang S et al: SOX2 plays a critical role in the pituitary, forebrain, and eye during human embryonic development. J Clin Endocrinol Metab 2008; 93: 1865-1873.

19 Kelberman D, Rizzoti K, Avilion A et al: Mutations within Sox2/SOX2 are associated with abnormalities in the hypothalamo-pituitary-gonadal axis in mice and humans. J Clin Invest 2006; 116: 2442-2455.

20 Sato $\mathrm{N}$, Kamachi $\mathrm{Y}$, Kondoh $\mathrm{H}$ et al: Hypogonadotropic hypogonadism in an adult female with a heterozygous hypomorphic mutation of SOX2. Eur J Endocrinol 2007; 156: $167-171$.

21 Mihelec M, Abraham P, Gibson K et al: Novel SOX2 partner-factor domain mutation in a four-generation family. Eur J Hum Genet 2009; 17: 1417-1422.

22 Zenteno JC, Perez-Cano HJ, Aguinaga M: Anophthalmia-esophageal atresia syndrome caused by an SOX2 gene deletion in monozygotic twin brothers with markedly discordant phenotypes. Am J Med Genet A 2006; 140: 1899-1903.

23 Chassaing N, Gilbert-Dussardier B, Nicot F et al: Germinal mosaicism and familial recurrence of a SOX2 mutation with highly variable phenotypic expression extending from AEG syndrome to absence of ocular involvement. Am J Med Genet A 2007; 143: 289-291.

24 Faivre L, Williamson KA, Faber V et al: Recurrence of SOX2 anophthalmia syndrome with gonosomal mosaicism in a phenotypically normal mother. Am J Med Genet $A$ 2006; 140: 636-639.

25 Schneider A, Bardakjian TM, Zhou J et al: Familial recurrence of SOX2 anophthalmia syndrome: phenotypically normal mother with two affected daughters. Am J Med Genet $A$ 2008; 146A: 2794-2798.

26 Zhou J, Kherani F, Bardakjian TM et al: Identification of novel mutations and sequence variants in the SOX2 and $\mathrm{CHX} 10$ genes in patients with anophthalmia/microphthalmia. Mol Vis 2008; 14: 583-592. 